\title{
Small Cell Carcinoma of the Tonsil Treated with Irinotecan and Cisplatin: A Case Report and Literature Review
}

\author{
Yuichi Segawa ${ }^{a}$ Torahiko Nakashima $^{a}$ \\ Hideki Shiratsuchi $^{a}$ Risa Tanaka ${ }^{b}$ Kenji Mitsugi ${ }^{b}$ \\ Shizuo Komune ${ }^{a}$ \\ a Department of Otorhinolaryngology, Graduate School of Medical Sciences, \\ Kyushu University, and ${ }^{b}$ Department of Internal Medicine, Hamanomachi \\ General Hospital, Fukuoka, Japan
}

\section{Key Words}

Small cell carcinoma $\cdot$ Tonsil $\cdot$ Irinotecan $\cdot$ Cisplatin

\begin{abstract}
We report a rare case of extrapulmonary small cell carcinoma arising in the palatine tonsil treated by combined chemotherapy with irinotecan/cisplatin following irradiation therapy. This chemotherapy regimen was recently found to be effective for small cell lung carcinoma. Our case is the first report of combined irinotecan/cisplatin chemotherapy to treat extrapulmonary small cell carcinoma of the oropharynx.
\end{abstract}

\section{Introduction}

Small cell carcinoma is a malignancy that occurs mainly in the lung, and primary lesions in the head and neck are very rare. Extrapulmonary small cell carcinoma (EPSCC) shows a fair response to either chemotherapy or chemoradiotherapy, similar to small cell lung carcinoma (SCLC); however, poor prognosis has been reported due to its high metastatic potential. Irinotecan hydrochloride (CPT-11), a topoisomerase I inhibitor, has been reported to be effective against SCLC [1]. Here, we report a rare case of EPSCC of the tonsil, treated with CPT-11 and cisplatin (CDDP), which is the recent treatment protocol for SCLC. The present case represents the first report of CPT-11 usage for EPSCC of the oropharynx. Our case suggests that CPT-11 and CDDP may become an effective treatment option for EPSCC of the oropharynx. 


\section{Case Report}

A 65-year-old male presented to our hospital complaining of sore throat for 2 weeks. He smoked heavily ( 70 cigarettes per day for 40 years) and was also a long-time alcohol drinker. His medical history included diabetes mellitus and aortic stenosis. Physical examination revealed an ulcerated mass in the left palatine tonsil and multiple swollen left neck lymph nodes. Computed tomography (CT) scans of the head and neck revealed a well-defined, $3.2 \times 3 \mathrm{~cm}$ mass in the left tonsil with multiple left cervical lymph node involvement.

A biopsy from the tonsillar mass was performed. Hematoxylin and eosin-stained sections revealed nests of monotonous, small, round carcinoma cells with scant cytoplasm and frequent mitotic figures in the subepithelial tissue (ig. 1). Immunohistochemically, the tumor cells were positive for neural cell adhesion molecule (CD56), synaptophysin, chromogranin, cytokeratin, and epithelial membrane antigen; the cells were negative for S-100 protein, HMB-45, leukocyte common antigen, and thyroid transcription factor-1 (fig. 2a, b). The MIB-1 labeling index was $88.5 \%$ (fig. 2c). These findings indicated small cell carcinoma.

To differentiate primary small cell carcinoma from a metastatic SCLC, a chest CT was performed, revealing no lesions. In addition, systemic workup, such as of the brain, as well as abdominal CT and FDG-PET showed negative findings for distant metastasis. The tumor was diagnosed as oropharyngeal cancer, T2N2bM0 (TNM classification, UICC, 2002).

Because cardiac echo revealed severe aortic stenosis and moderate aortic regurgitation, the patient received aortic valve replacement before treating the EPSCC of the tonsil. The tonsillar mass then grew to $4.6 \times 3.8 \mathrm{~cm}$, and the left neck lymph nodes also progressed ( $\underline{\text { fig. } 3}$ ); however, distant metastases were not recognized.

One month after the cardiac operation, he underwent radiotherapy of the tonsil and the bilateral cervical area with a dosage of $65.4 \mathrm{~Gy}$ in 35 fractions. He was also planned to receive three courses of chemotherapy with a regimen including CPT-11 $\left(60 \mathrm{mg} / \mathrm{m}^{2}\right.$, days 1 and 15$)$ and CDDP $\left(30 \mathrm{mg} / \mathrm{m}^{2}\right.$, days 1 and 15) at 4-week intervals. Because severe thrombocytopenia occurred after the first course of chemotherapy, the second and third courses were performed with reduced doses of CPT-11 (48 $\mathrm{mg} / \mathrm{m}^{2}$, days 1 and 15$)$ and CDDP (24 mg/m², days 1 and 15) at 3-week intervals. After chemo/radiotherapy, complete remission of the tumor was achieved.

Recurrent disease appeared in his liver 9 months after chemotherapy. The patient died from liver failure 2 years after the initial presentation.

\section{Discussion}

EPSCC compromises only 2.5-4\% of all small cell carcinomas and is commonly found in the esophagus, colon, pancreas, uterus, and breast. It reveals a fair response to either chemotherapy or chemoradiotherapy but exhibits a poor prognosis. In general, the clinical course of EPSCC is known to be aggressive because of frequent metastases and recurrences. Therefore, it should be considered a 'systemic' disease from the onset of treatment, even when no distant metastasis is recognized at the first medical examination.

In the head and neck region, the most common sites are the larynx, nose, and paranasal sinuses, and the major salivary glands [2]. Primary small cell carcinoma of the tonsil is rare; only 11 cases have been reported [3-9]. The prognosis of patients with EPSCC of the tonsil has been poor, as 6 of those 11 patients died from the disease mainly due to systemic metastasis.

When a diagnosis of EPSCC arising in the head and neck region is made, the patient should be evaluated first to determine whether the disease is a primary or metastatic 
cancer. Primary SCLC is rarely occult because it is aggressive and fast-growing. In our case, chest and abdominal CT and FDG-PET revealed no other lesions, and the tonsillar mass was determined to be the primary lesion.

The histopathological aspects of EPSCC include round, oval- to spindle-shaped small cells with dense chromatin, absence of nucleoli, and inconspicuous cytoplasm. Mitosis, necrosis, apoptosis, and lymphatic, vascular, and perineural invasion are common. Immunohistochemical staining for neuroendocrine markers, including chromogranin A, synaptophysin, and CD56, is usually positive. In this case, histologic features and immunoreactivity for neuroendocrine markers were suggestive of EPSCC.

Although the treatment of this tumor has not been defined clearly, it can require surgical excision of the localized tumor, radiation therapy to the primary site, multipledrug chemotherapy, or a combination of these modalities. The chemotherapeutic regimens for EPSCC are similar to those employed in SCLC. Platinum-based chemotherapy, such as the use of etoposide (VP-16) and CDDP, has been recognized as the standard regimen for EPSCC [2]. Recently, CPT-11 combined with CDDP was found to be more effective than VP-16 and CDDP [1]. Two cases of chemotherapy using CPT11 and CDDP have been reported for EPSCC arising in the head and neck region: one in the larynx and the other in the nasal cavity $[10,11]$. Both cases achieved long-term remission. In our case, radiation therapy to primary and locoregional sites followed by immediate chemotherapy with CPT-11/CDDP resulted in complete remission of the tumor, although the patient later died of liver metastasis.

An effective systemic chemotherapy should be established to regulate this highly metastatic disease.

\section{Conclusion}

In this report, we describe a case of small cell carcinoma of the tonsil treated by combined chemotherapy with CPT-11/CDDP after radiotherapy. This is the first report on the use of combined CPT-11/CDDP chemotherapy in small cell carcinoma of the oropharynx.

\section{Acknowledgement}

We thank KN International for revising the English used in this report. 


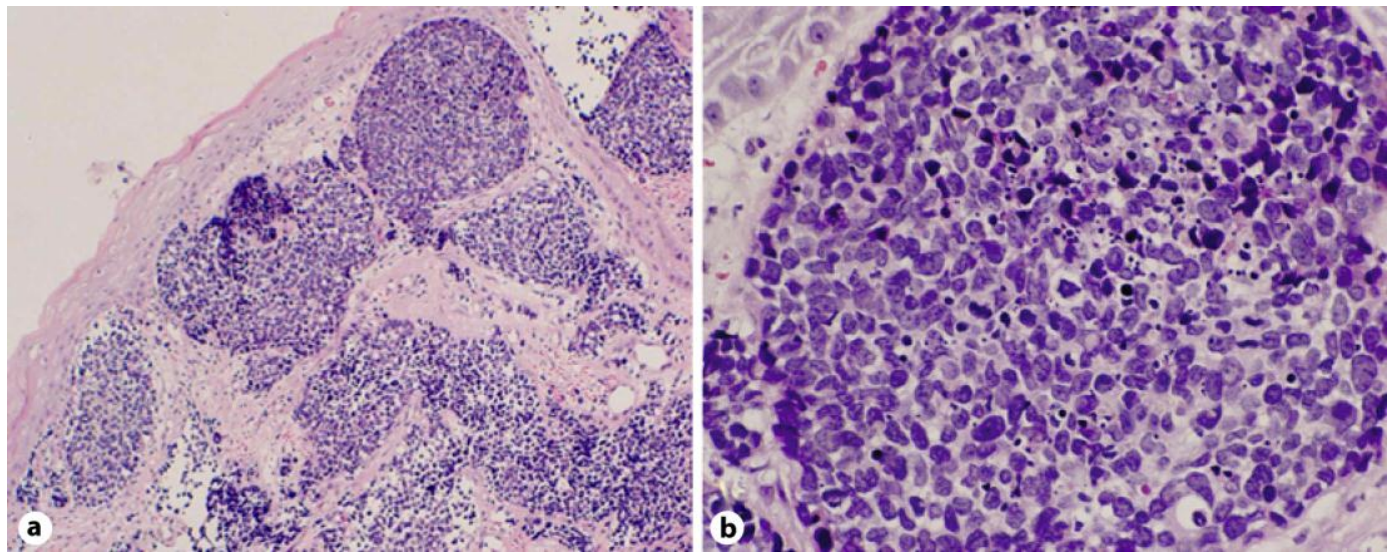

Fig. 1. Histologic appearance of the tumor. a The tumor was arranged in nests and grew in the subepithelial tissue. $\mathbf{b}$ The tumor had scant cytoplasm, and mitotic figures were frequently seen.
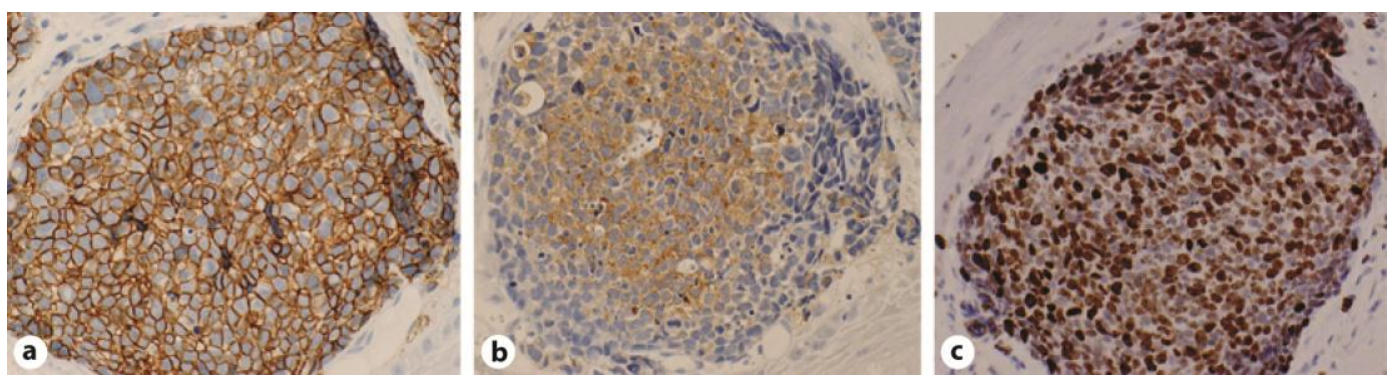

Fig. 2. Immunohistochemical staining of the tumor. a Staining of cell membrane for CD56.

b Cytoplasmic staining for synaptophysin. c Tumor staining reveals a high MIB-1 labeling index of $88.5 \%$. 

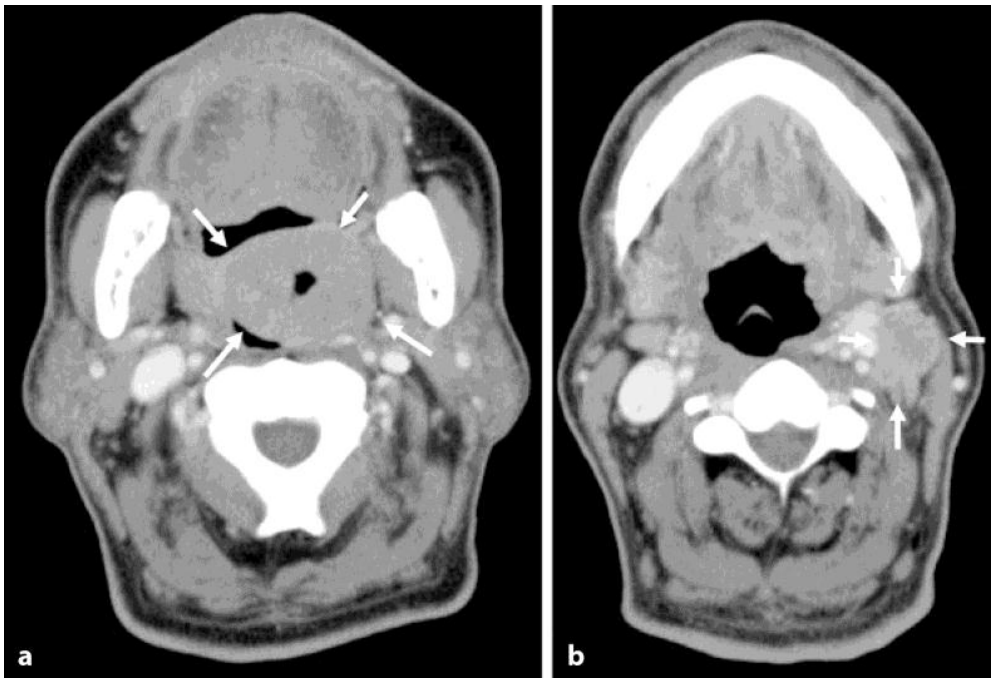

Fig. 3. Computed tomography. a Tumor of the left palatine tonsil with ulceration (arrows). b Swelling of the left cervical lymph nodes (arrows).

\section{References}

$\checkmark 1$ Noda K, Nishiwaki Y, Kawahara M, Negoro S, Sugiura T, Yokoyama A, Fukuoka M, Mori K, Watanabe K, Tamura T, Yamamoto S, Saijo N: Irinotecan plus cisplatin compared with etoposide plus cisplatin for extensive small-cell lung cancer. N Engl J Med 2002;346:85-91.

2 Renner G: Small cell carcinoma of the head and neck: a review. Semin Oncol 2007;34:3-14.

3 Koss LG, Spiro RH, Hajdu S: Small cell (oat cell) carcinoma of minor salivary gland origin. Cancer 1972;30:737-741.

4 Abedi E, Sismanis A: Extrapulmonary oat-cell carcinoma of the tonsil. Ear Nose Throat J 1987;66:112115.

5 Heimann R, Dehou MF, Lentrebecq B, Faverly D, Simonet ML, Dor P, Chanoine F: Anaplastic small cell (oat cell) carcinoma of the tonsils: report of two cases. Histopathology 1989;14:67-74.

-6 Bawa R, Wax MK: Small cell carcinoma of the tonsil. Otolaryngol Head Neck Surg 1995;113:328-333.

-7 Mineta H, Miura K, Takebayashi S, Araki K, Ueda Y, Harada H, Misawa K: Immunohistochemical analysis of small cell carcinoma of the head and neck: a report of four patients and a review of sixteen patients in the literature with ectopic hormone production. Ann Otol Rhinol Laryngol 2001;110:76-82.

8 Weng CT, Chu PY, Liu MT, Chen MK: Small cell carcinoma of the head and neck: a single institution's experience and review of the literature. J Otolaryngol Head Neck Surg 2008;37:788-793.

9 Hatoum GF, Patton B, Takita C, Abdel-Wahab M, LaFave K, Weed D, Reis IM: Small cell carcinoma of the head and neck: the University of Miami experience. Int J Radiat Oncol Biol Phys 2009;74:477-481.

10 Sone M, Uchida I, Tominaga M, Sugiura S, Nagasaka T, Nakashima T: Small cell carcinoma of the larynx treated with irinotecan and cisplatin. Auris Nasus Larynx 2006;33:223-225.

11 Hakuba N, Hyodo M, Yokoi T, Sato H: Irinotecan (CPT-11) combined with cisplatin for small cell carcinoma of the nasal cavity. Auris Nasus Larynx 2006;33:67-70. 